\title{
Development and validation of a preoperative nomogram for predicting survival of patients with locally advanced prostate cancer after radical prostatectomy
}

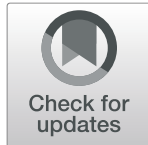

Xianghong Zhou ${ }^{1,2+}$, Qingyang Ning ${ }^{1,3+}$, Kun Jin², Tao Zhang ${ }^{3}$ and Xuelei Ma ${ }^{1{ }^{*+}}$ (1)

\begin{abstract}
Background: For selected locally advanced prostate cancer (PCa) patients, radical prostatectomy (RP) is one of the first-line treatments. We aimed to develop a preoperative nomogram to identify what kinds of patients can get the most survival benefits after RP.

Methods: We conducted analyses with data from the Surveillance, Epidemiology, and End Results (SEER) database. Covariates used for analyses included age at diagnosis, marital status, race, American Joint Committee on Cancer (AJCC) 7th TNM stage, Prostate specific antigen, Gleason biopsy score (GS), percent of positive cores. We estimated the cumulative incidence function for cause-specific death. The Fine and Gray's proportional subdistribution hazard approach was used to perform multivariable competing risk analyses and reveal prognostic factors. A nomogram was built by these factors (including GS, percent of positive cores and $\mathrm{N}$ stage) and validated by concordance index and calibration curves. Risk stratification was established based on the nomogram.

Results: We studied 14,185 patients. N stage, GS, and percent of positive cores were the independent prognostic factors used to construct the nomogram. For validating, in the training cohort, the C-index was 0.779 (95\% Cl 0.736-0.822), and in the validation cohort, the C-index was 0.773 ( $95 \% \mathrm{Cl} 0.710-0.836$ ). Calibration curves showed that the predicted survival and actual survival were very close. The nomogram performed better over the AJCC staging system (C-index 0.779 versus 0.764 for training cohort, and 0.773 versus 0.744 for validation cohort). The new stratification of risk groups based on the nomogram also showed better discrimination than the AJCC staging system.
\end{abstract}

Conclusions: The preoperative nomogram can provide favorable prognosis stratification ability to help clinicians identify patients who are suitable for surgery.

Keywords: Prostate cancer, Radical prostatectomy, Nomogram

\footnotetext{
* Correspondence: drmaxuelei@gmail.com

†Xianghong Zhou, Qingyang Ning and Xuelei Ma contributed equally to this work.

'Department of Biotherapy, West China Hospital and State Key Laboratory of Biotherapy, Sichuan University, Chengdu, People's Republic of China Full list of author information is available at the end of the article
}

(c) The Author(s). 2020 Open Access This article is distributed under the terms of the Creative Commons Attribution 4.0 International License (http://creativecommons.org/licenses/by/4.0/), which permits unrestricted use, distribution, and reproduction in any medium, provided you give appropriate credit to the original author(s) and the source, provide a link to the Creative Commons license, and indicate if changes were made. The Creative Commons Public Domain Dedication waiver (http://creativecommons.org/publicdomain/zero/1.0/) applies to the data made available in this article, unless otherwise stated. 


\section{Background}

Prostate cancer ( $\mathrm{PCa}$ ) is one of the most common malignant tumors of males around the world, and it is estimated that there will be 174,650 new male cases in the United States in 2019 [1]. The European Association of Urology defined PCa with cT3-4 or $\mathrm{N}+$ as locally advanced PCa [2]. Patients with locally advanced PCa have an increased risk of disease progression and cancerspecific death [2]. Due to many factors like the American national guidelines advising against PSA screening, increasing applications for active surveillance, etc., the proportion of men diagnosed with locally advanced PCa has increased [3, 4]. Nowadays, the standard treatment of locally advanced PCa is still unclear due to the absence of level 1 evidence. For selected locally advanced PCa patients, radical prostatectomy (RP) is one of the first-line treatments recommended by the guidelines [2, 5]. However, it is still controversial as to which types of patients could get the most survival benefits from RP.

Some studies about the risk stratification of patients with high-risk PCa (including locally advanced PCa) after RP had been reported. Previous researchers performed a retrospective analysis of 315 high-risk PCa patients after RP in their hospital. They selected biochemical progression as the primary endpoint, and reported that the risk of biochemical progression of high-risk PCa after RP could be stratified by Gleason Score (GS) at biopsy $(\geq 8)$ and \% positive core ( $\geq 30 \%$ ) [6]. Another research team studied 813 high-risk patients undergoing RP, and found 3 preoperative criteria including stage $\mathrm{CT} 2$ or greater, prostate specific antigen $(\mathrm{PSA})>20 \mathrm{ng} / \mathrm{mL}$, and $\mathrm{GS}>8$. In their study, the number of meeting preoperative criteria was significantly predictive for recurrence-free survival (RFS), and overall survival (OS) [7]. Although these studies had proposed their own risk stratification models for high-risk PCa patients undergone RP, the patients involved in these studies were not fully compliant with the current more common definition of locally advanced $\mathrm{PCa}$, the impact of prognostic factors on patient survival outcome was not quantitative, the weight between the various prognostic factors was not clear enough and the cohorts were not large enough.

To circumvent these defects, we evaluated the prostate cancer-specific survival (CSS) at a large cohort to assess potential preoperative prognostic factors, and developed a preoperative nomogram. This nomogram could be used to predict the CSS of patients with locally advanced PCa after RP and to identify what kinds of patients can get the most survival benefits from RP.

\section{Methods}

\section{Patient selection}

The Surveillance, Epidemiology, and End Results (SEER) database is a free dataset made up of 18 population- based cancer registries. It releases cancer patients' general information annually and has almost covered $25 \%$ population of the United States [8].

From the SEER database, patients with a diagnosis of adenocarcinoma of the prostate (International Classification of diseases-O-3 code: C61.9) between 2010 and 2016 were selected. The TNM stages were assessed by the 7th edition of American Joint Committee on Cancer [AJCC] Cancer Staging Manual [9]. Inclusion and exclusion criteria were shown in the flowchart in detail (Fig. 1). All the included patients were randomly divided into the training cohort and validation cohort with a ratio of $7: 3$.

\section{Variables and endpoint}

Data for each patient were extracted from the SEER database including age at diagnosis, marital status, race, AJCC 7th TNM stage, PSA, Gleason biopsy score (GS), percent of positive cores at biopsy (\% positive core), and follow-up information. Age was categorized as $\leq 49$ years, $50-59$ years, $60-69$ years, and $\geq 70$ years. PSA was categorized as $\leq 10 \mathrm{ng} / \mathrm{mL}, 10-20 \mathrm{ng} / \mathrm{mL}$ (not including 10), and $>20 \mathrm{ng} / \mathrm{mL}$. GS was classified as $\leq 6,7(3+4), 7(4+$ $3)$, 8 , and $\geq 9$. \% positive core was classified as $0-25,25-$ $50 \%$ (not including 25\%), 50-75\% (not including 50\%), and $75-100 \%$ (not including 75\%). In addition to followup information, all clinical factors were obtained before undergoing RP. Due to the limitation of the SEER database, the information about detail procedures used to determine the lymph node positive was not been provided [10].

CSS was used as the primary endpoint. CSS was measured by all deaths caused by prostate cancer, complications of treatments, or unknown processes in patients with active prostate cancer. Follow-up time was defined as the time between the first treatment and the patient's death or last follow-up.

\section{Statistical analysis}

A $X^{2}$ test was used to expose the difference between the training cohort and validation cohort in categorical variables, and the results were presented as the frequency with its proportion. Kaplan-Meier method and log-rank test were used to expose each potential prognostic variable. Variables in the univariate analysis with $p$-value $<0.05$ were chosen for multivariate Cox proportional hazard regression to identify the independent prognostic factors. In addition, many patients with locally advanced PCa are the elderly with many comorbidities. Thus, there is a high risk of non-cancer competing risk events leading to death in the long-term survival [11]. In order to make the research more precise, we further implemented competing risk analyses. We estimated the cumulative incidence function (CIF) for cause-specific death, and tested the differences 


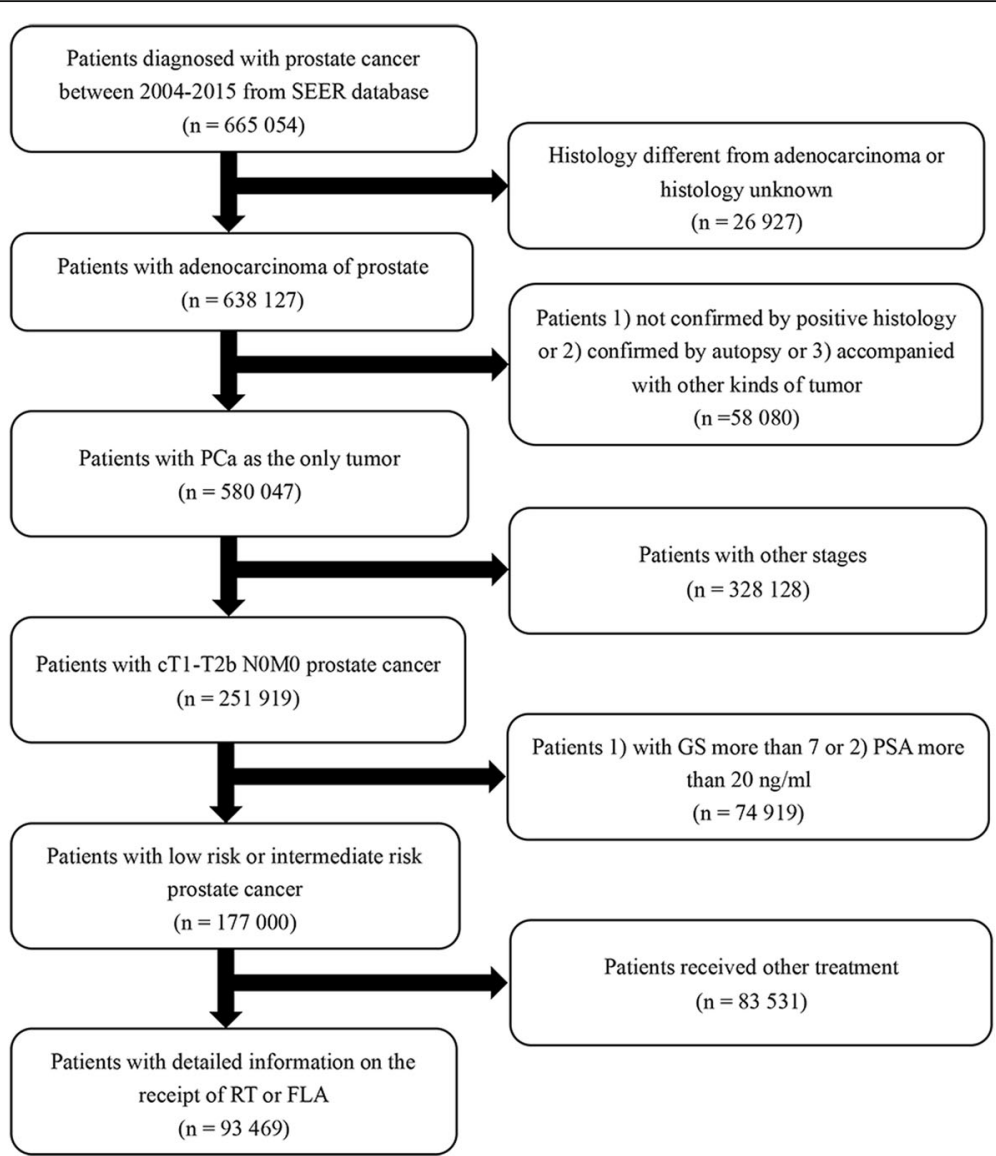

Fig. 1 Flowchart describing the selection of patients in the Surveillance, Epidemiology, and End Results (SEER) database, 2010-2016

by Gray's test to expose each prognostic variable. The Fine and Gray's proportional subdistribution hazard approach was used to conduct competing risk multivariable analyses. Based on clinical significance and $p$-value $<0.05$ in competing risk multivariable analyses, these independent prognostic factors were selected to construct a nomogram to predict 5-year cancer-specific mortality (CSM) probabilities of patients.

The performance of the nomogram was validated by measuring discrimination and calibration in both training set and validation set. The discrimination of the nomogram was assessed by $\mathrm{C}$-index (concordance index). The value of C-index ranged from 0.5 (no discrimination) to 1 (perfect discrimination), and higher C-index showed better discrimination of the prognostic model [12]. By comparing the plot of predicted probabilities from the nomogram with the plot of actual probabilities of CSS, the calibration curve was used to compare the predicted survival using nomogram with the actual observed survival, with 1000 resamples of bootstrapping. In addition, we conducted a risk stratification of the nomogram total risk score. The cut-off values were determined using X-tile software and by the minimal $p$-value approach [13]. The discrimination abilities between the nomogram and AJCC staging system were compared by $\mathrm{C}$-index and Kaplan-Meier curve.

The statistical software packages $R$ (The $R$ Foundation) and $\mathrm{R}$ packages cmprsk, rms, survival, mstate, and regplot were used in the above statistical analyses. A $p$ value $<0.05$ was considered statistically significant.

\section{Results}

\section{Patient characteristics}

A total of 14,185 eligible patients were included in the current analysis as the primary cohort. Among all patients, 9932 patients were placed within the training cohort, and 4253 patients were placed within the validation cohort randomly. Detail patients' characteristics were shown in Table 1 . The median follow-up was 43 months (95\% CI 42-44 months). There were no statistically significant differences in baseline characteristics between the training cohort and the validation cohort.

\section{Determination of independent preoperative prognostic factors}

The Fine and Gray's proportional subdistribution hazard approach was performed to demonstrate the independent 
Table 1 Descriptive characteristics of 14,185 locally advanced prostate cancer patients undergoing radical prostatectomy between 2010 and 2016 from the Surveillance Epidemiology and End Results database. Primary 14,185 patients were randomly divided into 2 cohorts: training cohort and validation cohort

\begin{tabular}{|c|c|c|c|c|c|c|c|}
\hline \multirow[t]{2}{*}{ Variable } & \multicolumn{2}{|c|}{$\begin{array}{l}\text { Primary Cohort } \\
(n=14,185)\end{array}$} & \multicolumn{2}{|c|}{$\begin{array}{l}\text { Training Cohort } \\
(n=9932)\end{array}$} & \multicolumn{2}{|c|}{$\begin{array}{l}\text { Validation Cohort } \\
(n=4253)\end{array}$} & \multirow[t]{2}{*}{$P$-value } \\
\hline & Number & $\%$ & Number & $\%$ & Number & $\%$ & \\
\hline Age & & & & & & & 0.131 \\
\hline$<50$ & 514 & 3.6 & 356 & 3.6 & 158 & 3.7 & \\
\hline $50-59$ & 4092 & 28.9 & 2907 & 29.3 & 1185 & 27.9 & \\
\hline $60-69$ & 7460 & 52.6 & 5163 & 52.0 & 2297 & 54.0 & \\
\hline$>69$ & 2119 & 14.9 & 1506 & 15.2 & 613 & 14.4 & \\
\hline Race & & & & & & & 0.658 \\
\hline White & 11,445 & 80.7 & 8033 & 80.9 & 3412 & 80.2 & \\
\hline Black & 1852 & 13.0 & 1282 & 12.9 & 570 & 13.4 & \\
\hline Other ${ }^{a}$ & 888 & 6.3 & 617 & 6.2 & 271 & 6.4 & \\
\hline Marital Status & & & & & & & 0.501 \\
\hline Married & 10,429 & 73.5 & 7289 & 73.4 & 3140 & 73.8 & \\
\hline Single $e^{b}$ & 3001 & 21.2 & 2100 & 21.1 & 901 & 21.2 & \\
\hline Unknown & 755 & 5.3 & 543 & 5.5 & 212 & 5.0 & \\
\hline T stage & & & & & & & 0.019 \\
\hline $\mathrm{T} 1-2$ & 290 & 2.0 & 185 & 1.9 & 105 & 2.5 & \\
\hline T3-4 & 13,895 & 98.0 & 9747 & 98.1 & 4148 & 97.5 & \\
\hline N stage & & & & & & & 0.077 \\
\hline NO & 12,215 & 86.1 & 8586 & 86.4 & 3629 & 85.3 & \\
\hline $\mathrm{N} 1$ & 1970 & 13.9 & 1346 & 13.6 & 624 & 14.7 & \\
\hline PSA level (ng/ml) & & & & & & & 0.012 \\
\hline$\leq 10$ & 9447 & 66.6 & 6682 & 67.3 & 2765 & 65.0 & \\
\hline $10-20$ & 3043 & 21.5 & 2066 & 20.8 & 977 & 23.0 & \\
\hline$>20$ & 1695 & 11.9 & 1184 & 11.9 & 511 & 12.0 & \\
\hline GS biopsy & & & & & & & 0.402 \\
\hline$\leq 6$ & 2142 & 15.1 & 1510 & 15.2 & 632 & 14.9 & \\
\hline $7(3+4)$ & 4571 & 32.2 & 3234 & 32.6 & 1337 & 31.4 & \\
\hline $7(4+3)$ & 3015 & 21.3 & 2080 & 20.9 & 935 & 22.0 & \\
\hline 8 & 2436 & 17.2 & 1684 & 17.0 & 752 & 17.7 & \\
\hline$\geq 9$ & 2021 & 14.2 & 1424 & 14.3 & 597 & 14.0 & \\
\hline$\%$ positive core biopsy & & & & & & & 0.638 \\
\hline $00-25 \%$ & 2986 & 21.1 & 2088 & 21.0 & 898 & 21.1 & \\
\hline $25-50 \%$ & 4982 & 35.1 & 3515 & 35.4 & 1467 & 34.5 & \\
\hline $50-75 \%$ & 3008 & 21.2 & 2081 & 21.0 & 927 & 21.8 & \\
\hline $75-100 \%$ & 3209 & 22.6 & 2248 & 22.6 & 961 & 22.6 & \\
\hline AJCC staging system & & & & & & & 0.140 \\
\hline$\| I B$ & 10,820 & 76.3 & 7591 & 76.4 & 3229 & 75.9 & \\
\hline$\| I I C$ & 1395 & 9.8 & 995 & 10.0 & 400 & 9.4 & \\
\hline IVA & 1970 & 13.9 & 1346 & 13.6 & 624 & 14.7 & \\
\hline
\end{tabular}

Abbreviation: AJCC American Joint Committee on Cancer

${ }^{a}$ Other: American Indian/AK Native, Asian/Pacific Islande

${ }^{\mathrm{b}}$ Single: Divorced, Separated, Single (never married), Widowed, unmarried 
Table 2 Univariate analyses, multivariate analyses of preoperative prognostic factors influencing cancer-specific survival outcomes in the training cohort

\begin{tabular}{|c|c|c|c|c|c|c|}
\hline Variable & $\begin{array}{l}\text { Univariate analyses } \\
\text { (KM) } p \text {-value }\end{array}$ & $\begin{array}{l}\text { Univariate analyses } \\
\text { (CIF) } p \text {-value }\end{array}$ & $\begin{array}{l}\text { Multivariate Cox regression } \\
\text { analyses HR }(95 \% \mathrm{Cl})\end{array}$ & $P$-value & $\begin{array}{l}\text { Multivariate Competing risk } \\
\text { analyses sdHR }(95 \% \mathrm{Cl})\end{array}$ & $P$-value \\
\hline Age & 0.041 & 0.047 & & & & \\
\hline$<50$ & & & Ref. & & Ref. & \\
\hline $50-59$ & & & $1.111(0.440-2.804)$ & 0.824 & $1.110(0.440-2.802)$ & 0.826 \\
\hline $60-69$ & & & $0.805(0.321-2.0203)$ & 0.645 & $0.803(0.320-2.015)$ & 0.641 \\
\hline$>69$ & & & $1.291(0.495-3.373)$ & 0.602 & $1.284(0.492-3.355)$ & 0.609 \\
\hline Race & 0.860 & 0.852 & & & & \\
\hline White & & & Ref. & & Ref. & \\
\hline Black & & & $0.988(0.587-1.661)$ & 0.962 & $0.987(0.587-1.661)$ & 0.962 \\
\hline Other & & & $0.961(0.487-1.898)$ & 0.908 & $0.960(0.486-1.897)$ & 0.907 \\
\hline Marital Status & 0.790 & 0.815 & & & & \\
\hline Married & & & Ref. & & Ref. & \\
\hline Single & & & $1.004(0.675-1.495)$ & 0.984 & $1.003(0.674-1.493)$ & 0.988 \\
\hline Unknown & & & $1.193(0.602-2.364)$ & 0.613 & $1.189(0.600-2.357)$ & 0.619 \\
\hline T stage & 0.300 & 0.304 & & & & \\
\hline $\mathrm{T} 1-2$ & & & Ref. & & Ref. & \\
\hline T3-4 & & & $6.511(0.897-47.269)$ & 0.064 & $6.493(0.895-47.142)$ & 0.064 \\
\hline $\mathrm{N}$ stage & $<0.001$ & $<0.001$ & & & & \\
\hline NO & & & Ref. & & Ref. & \\
\hline N1 & & & $2.431(1.695-3.489)$ & $<0.001$ & $2.429(1.693-3.483)$ & $<0.001$ \\
\hline PSA level (ng/ml) & $<0.001$ & $<0.001$ & & & & \\
\hline$\leq 10$ & & & Ref. & & Ref. & \\
\hline $10-20$ & & & $1.141(0.765-1.702)$ & 0.519 & $1.140(0.764-1.701)$ & 0.521 \\
\hline$>20$ & & & $1.472(0.962-2.253)$ & 0.075 & $1.473(0.962-2.253)$ & 0.075 \\
\hline GS biopsy & $<0.001$ & $<0.001$ & & & & \\
\hline$\leq 6$ & & & Ref. & & Ref. & \\
\hline $7(3+4)$ & & & $4.252(1.277-14.152)$ & 0.018 & $4.253(1.2779-14.157)$ & 0,018 \\
\hline $7(4+3)$ & & & $6.096(1.827-20.338)$ & 0.003 & $6.099(1.828-20.349)$ & 0.003 \\
\hline 8 & & & $9.796(2.972-32.286)$ & $<0.001$ & $9.8068(2.975-32.322)$ & $<0.001$ \\
\hline$\geq 9$ & & & $18.879(5 . .88-61.576)$ & $<0.001$ & $18.888(5.791-61.605)$ & $<0.001$ \\
\hline$\%$ positive core biopsy & $<0.001$ & $<0.001$ & & & & \\
\hline $00-25 \%$ & & & Ref. & & Ref. & \\
\hline $25-50 \%$ & & & 1.099 (0.599-2.018) & 0.760 & $1.100(0.599-2.020)$ & 0.758 \\
\hline $50-75 \%$ & & & $1.145(0.604-2.173)$ & 0.678 & $1.145(0.604-2.173)$ & 0.678 \\
\hline $75-100 \%$ & & & $2.176(1.219-3.8837)$ & 0.009 & $2.174(1.218-3.881)$ & 0.009 \\
\hline
\end{tabular}

Abbreviation: KM Kaplan-Meier method, CIF Cumulative incidence function, sdHR Subdistribution hazard ratio, Ref. Reference, \% positive core biopsy Percent of positive cores at biopsy

preoperative prognostic factors in the training cohort. Table 2 showed the detail univariate and multivariate analysis results. In the univariate analyses, age, $\mathrm{N}$ stage, PSA, GS, \% positive core biopsy were variables with significant impact on CSS.

In the Fine and Gray's proportional subdistribution hazard approach, N stage, GS, \% positive core biopsy remained significantly, while age and PSA did not show significant impact on CSS. These significant variables were thought as independent prognostic factors of CSS for locally advanced PCa patients after RP.

\section{Construction and validation of the nomogram}

Using the independent prognostic factors including $\mathrm{N}$ stage, GS, and \%positive core biopsy, a nomogram was constructed to predict each locally advanced $\mathrm{PCa}$ 


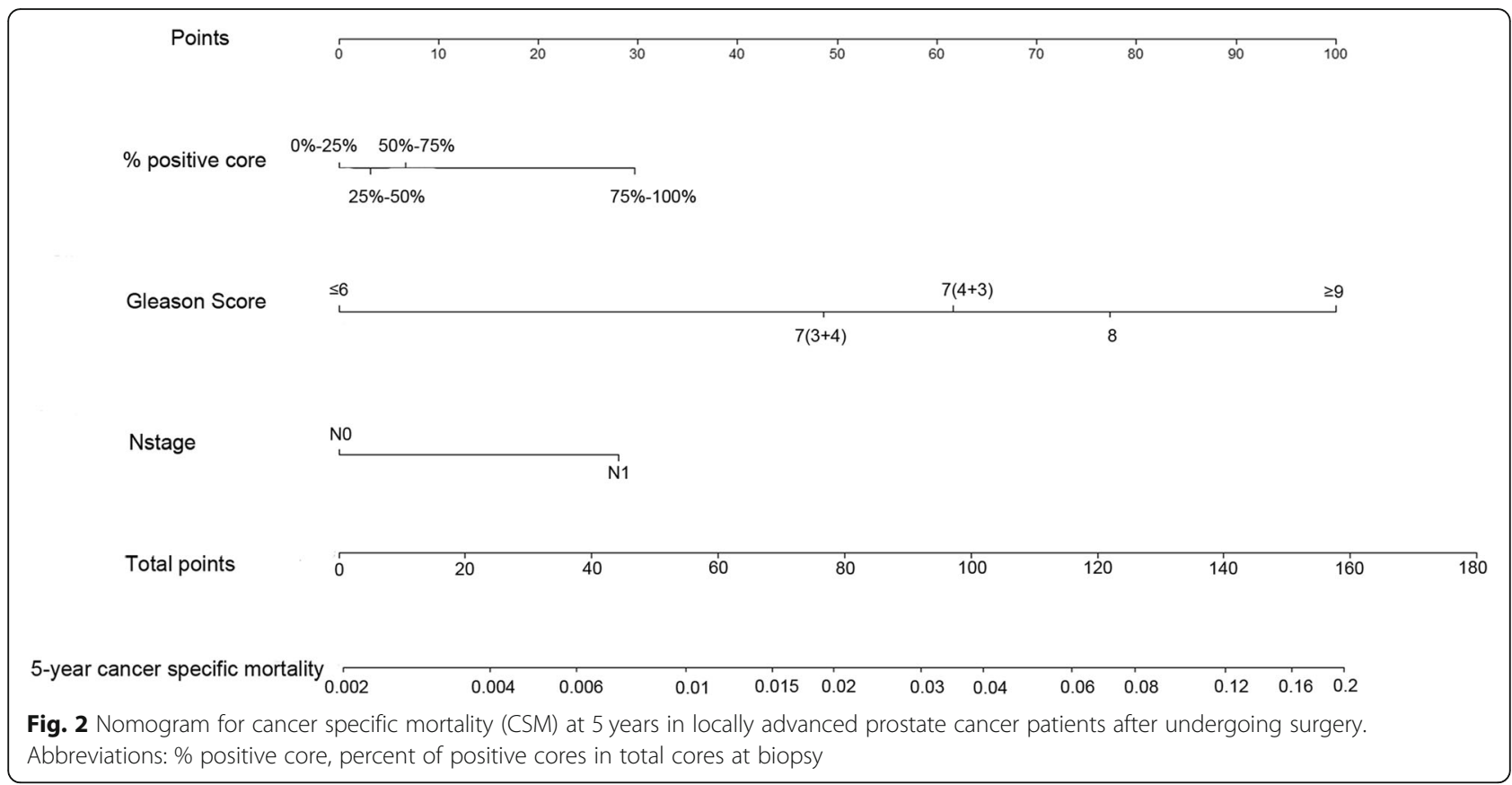

patient's probability of CSM at 5 years after RP (Fig. 2).

The nomograms were validated using $\mathrm{C}$-index and calibration curve. In the training cohort, the $\mathrm{C}$-index was 0.779 (95\% CI $0.736-0.822)$. In the validation cohort, the C-index was 0.773 (95\% CI 0.710-0.836). The C-index values indicated good discrimination of the nomogram (>0.7). The calibration curves showed good agreement between prediction by nomogram and actual 5 -year CSS in both training cohort and validation cohort (Fig. 3).

\section{Definition of nomogram risk group stratification}

The score corresponding to each nomogram variable was listed in detail in Table 3. By summing the score for each nomogram variable, we got the nomogram total risk score for each patient in both the training cohort and the validation cohort. The patients were divided into three nomogram risk groups for CSS: a low-risk group with 0-77 points, a middle-risk group with 79-108 points, a high-risk group with no less than 112 points.

\section{Comparison of nomogram with AJCC staging system}

We compared the nomogram with the 8th edition AJCC staging system. In the training cohort, the C-index of AJCC staging system was 0.764 (95\% CI 0.719-0.809), and the C-index of the nomogram was 0.779 (95\% CI 0.736-0.822). In the validation cohort, the C-index of AJCC staging system was 0.744 (95\% CI 0.707-0.817), and the C-index of the nomogram was 0.773 (95\% CI $0.710-0.836)$. In both cohorts, the nomogram performed
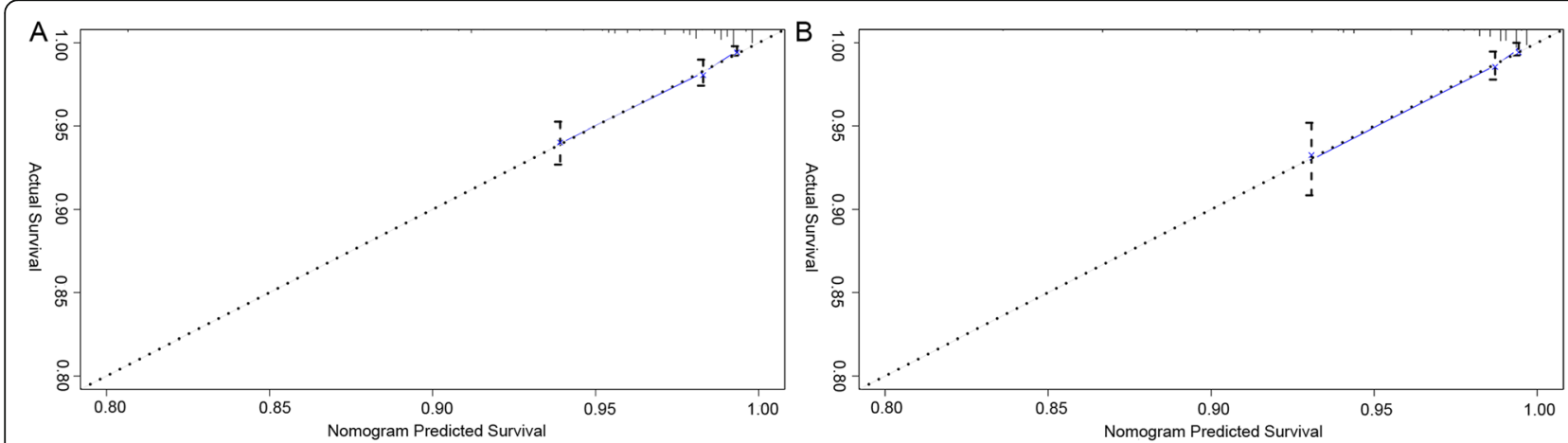

Fig. 3 Calibration curves for cancer specific survival (CSS) at 5 years in locally advanced prostate cancer patients after undergoing surgery in the training cohort (a) and the validation cohort (b). The horizontal axis is the survival rate predicted by the nomogram, and the vertical axis is the actual survival rate. The dashed line indicates the predicting survival rate completely fits the actual survival rate 
Table 3 Detailed risk scores of all prognostic factors in the nomogram

\begin{tabular}{ll}
\hline Variables & Nomogram risk score \\
N stage & 0 \\
N0 & 28 \\
N1 & \\
Gleason Score & \\
$\leq 6$ & 49 \\
$7(3+4)$ & 62 \\
$7(4+3)$ & 77 \\
8 & 100 \\
$\geq 9$ & 0 \\
Percent of positive cores \\
$0-25 \%$ & 3 \\
$25-50 \%$ & 7 \\
$50-75 \%$ & 30 \\
$75-100 \%$ & Predicted probability of 5-year CSM at 5 years \\
Total points & $0.20 \%$ \\
0 & $0.64 \%$ \\
40 & $2.10 \%$ \\
80 & $6.75 \%$ \\
120 & $20.52 \%$ \\
160 &
\end{tabular}

Abbreviation: CSM Cancer specific mortality

better in C-index, indicating that the nomogram had a better discrimination ability than AJCC staging system.

The AJCC staging could divide the whole eligible patients in current study into 3 groups including IIIB, IIIC, and IVA. Relatively, as mentioned above, the risk groups based on the nomogram included low-risk group, middle-risk group, high-risk group. For risk groups based on the nomogram, the degree of separation of CIF curves of CSM between groups was more obvious than AJCC staging system in both training cohort and validation cohort (Fig. 4). The CIF curves still demonstrated the new stratification of risk groups based on the nomogram had better prognostic discrimination than AJCC staging system.

\section{Discussion}

Using a large cohort of 14,185 patients from the SEER database, we successfully used 3 independent preoperative prognostic factors to established a nomogram for predicting 5-year CSM for patients with locally advanced PCa. To our knowledge, this is the first preoperative predictive nomogram and nomogram-based risk group stratification built for patients with locally advanced PCa. At the same time, the patient samples are the latest relatively (2010-2016), which is conducive to reducing the impact of defects in pathology, surgery and laboratory testing techniques on the results. This nomogram and nomogram-based risk group stratification is not inferior or even better than the current AJCC staging system in terms of discrimination, and is more quantitative and intuitive, which is convenient for clinicians to use.

Nomograms use a variety of biological and clinical variables to graphically depict the probability that a clinical event may occur for each individual [14]. One of their unique abilities is to estimate individualized risk based on patient and disease characteristics in a graphical and user-friendly form. Nomograms can include many continuous variables and key factors of disease into the prognosis, and taking weights of each variable into account, to make predictive models more practical [14, 15]. Compared with the current prognostic prediction system like AJCC staging system, the nomogram showed advantages in many studies on different cancers [16, 17]. They can also establish individualized risk stratification and help clinicians identify suitable patients for optimal managements, and good results had been obtained in many studies of different tumor types [18-20].

In our study, the statistical analysis highlighted 3 preoperative prognostic factors including clinical $\mathrm{N}$ stage, GS, and \% positive core. Among them, GS had the greatest contribution to 5-year cancer-specific death (GS shared the most proportion of the nomogram total risk score.). GS is one of the most universal prognostic factors for PCa. International Society of Urological Pathology (ISUP) proposed in 2014, GS can be divided into five groups $(2-6,7(3+4), 7(4+3), 8, \geq 9)$ according to the differences of prognosis [21]. In our study, the classification of GS also referred to the ISUP grading system, and the risk trend shown by the nomogram was consistent with this grading system. Some previous studies had shown the relationship between the prognosis of PCa and GS $[6,22,23]$. These studies had confirmed the increased possibility of adverse clinical events including death and biochemical recurrence in PCa patients with increasing GS. But these studies did not show what the weight of GS compared to other variables on affecting patients was. \% positive core showed the obvious impact on CSS for patients in the current study, only inferior to GS. \% positive core refers to the proportion of positive needles in the needle biopsy to the total number of needles. Many studies had revealed the prognostic value of $\%$ positive core for $\mathrm{PCa}[6,22$, $24,25]$. In our nomogram, the risk score of group 75$100 \%$ increased significantly from 7 (group 50-75\%) to 30 , suggesting that $75-100 \%$ positive core can more significantly affect the patient's prognosis. This finding was similar to the results of a study of 195 patients with high-risk PCa who undergoing RP. In that study, 70\% positive was shown to have significant prognostic 

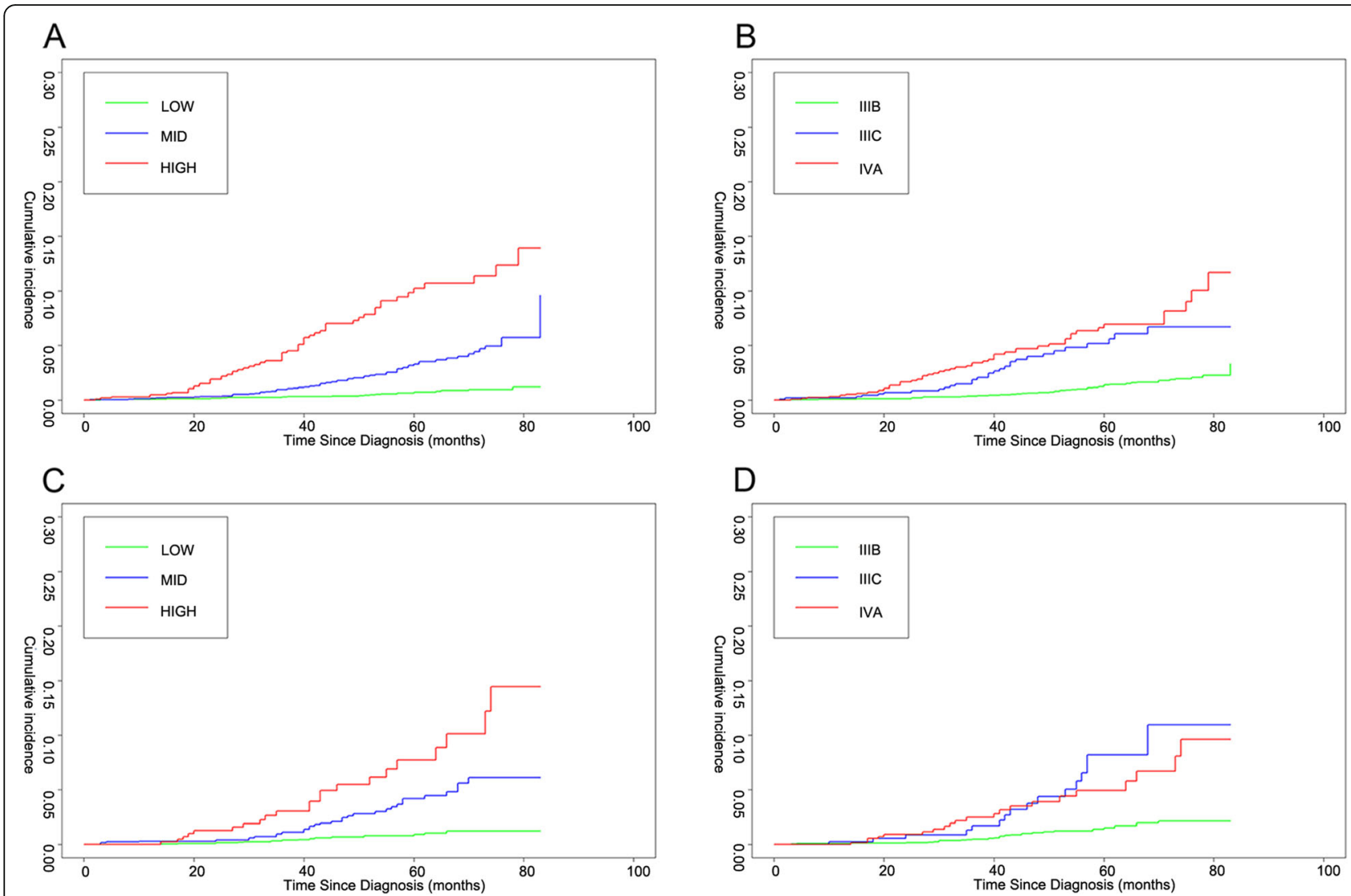

Fig. 4 Cumulative incidence function (CIF) curves of different risk stratification systems for cancer specific mortality (CSM) in locally advanced prostate cancer patients after undergoing surgery. Nomogram risk stratification and AJCC staging system were used in the training cohort (a, b) and validation cohort (c, d)

discrimination [24]. From the nomogram, regional lymph node metastasis (N1) was another important independent prognostic factor for CSS of locally advanced $\mathrm{PCa}$ patients. Previous studies had demonstrated that lymph node metastasis in most patients with PCa was associated with progressive disease, which was important for the prognosis of patients [26, 27]. A retrospective study of 229 patients indicated that the 5year disease-free survival of rate of patients with regional lymph node metastasis could be decreased from $85 \%$ to approximately $50 \%$ [28].

Relatively, the 8th AJCC staging system is currently a general PCa risk stratification system, not aim at specific disease stages and treatment therapies [29]. We compared our nomogram risk group stratification with AJCC staging system, and the results showed that our stratification had advantages in C-index ( 0.779 versus 0.763 for training cohort, and 0.773 versus 0.745 for validation cohort). At the same time, the nomogram risk stratification's CIF curve showed a better separation trend in both the training cohort and the validation cohort. There may be several reasons. First, our nomogram risk stratification considered the effect of $\%$ positive core on
CSS of locally advanced PCa patients after RP, compared to AJCC staging system. AJCC staging system only took GS and clinical N stage into account, but this study and other studies mentioned above confirmed the importance of \% positive core. Second, AJCC staging system was not quantitative, and the difference between the effects of GS and clinical N stage on prognosis was not reflected. In our nomogram risk stratification, we used different risk scores to reflect the weight of different prognostic factors, showing the obvious importance of GS among the three prognostic factors. At the same time, such quantitative and graphical tool is also easy for clinicians to use as manuals. In addition, the patients included in our study were more targeted than those in AJCC staging system after filtered by locally advanced $\mathrm{PCa}$ and RP. By classifying the prognosis of each patient after surgery, clinicians could better choose whether to perform surgery for each patient.

The main significance of the risk stratification proposed by us is to reveal the potential value of the three prognostic factors for the selection of locally advanced patients to receive RP and the influence weight of each factor on the prognosis of these patients. According to 
the latest guidelines, radical treatments including RP and RT are still the first-line treatments for locally advanced PCa. Only those patients who are unwilling or unable to receive any form of local treatment, or whose PSA growth is fast, or whose tumor differentiation is poor, are offered androgen deprivation therapy (ADT) monotherapy. The guidelines suggest that RP should be used in highly selective locally advanced PCa patients [30]. But at present, there is no high-level evidence on the appropriate selection criteria of RP or RT and the survival benefits comparison between RP and RT for locally advanced $\mathrm{PCa}$ patients. In our study, the risk stratification proposed by the nomogram provided a reference for the selection criteria of RP. Considering the difference of postoperative CSS, patients in highrisk group may be not suitable for RP, while patients in low-risk group may be the appropriate population for RP. For those who are not suitable for RP, the RT + ADT therapy may bring more survival benefits. In fact, previous studies have shown that RT + ADT therapy can bring significant benefits to locally advanced $\mathrm{PCa}$ patients with $\mathrm{pN}+[31,32]$. However, they didn't compare RP and RT directly. More clinical studies are needed to explore the most reasonable criteria for the selection of locally advanced $\mathrm{PCa}$ patients receiving RP or RT, especially the double arm randomized controlled studies of RP and RT, such as the SPCG-15 currently in progress [33].

There are still several limitations to our study. First, our research is constructed by retrospective data. Therefore, there may be some undetected potential bias factors in the study. Second, due to the SEER database's limitations, we lack functional data and disease progression data for further research. Third, we also lack additional independent external validation sets, and this is our important work goal in the future.

\section{Conclusions}

By analyzing a large cohort of 14,185 patients, we successfully revealed 3 independent preoperative prognostic factors for CSS of locally advanced PCa patients after RP. Using these 3 factors, we constructed and validated a preoperative prognostic nomogram that could predict the probability of CSM at 5 years of this type of patients after undergoing RP. Based on the nomogram, a risk stratification system was established to help clinicians better identify patients suitable for surgery.

\section{Abbreviations}

ADT: Androgen deprivation therapy; AJCC: American Joint Committee on Cancer; Cl: Confidence interval; CIF: Cumulative incidence function; CSM: Cancer-specific mortality; CSS: Cancer-specific survival; GS: Gleason score; OS: Overall survival; PCa: Prostate cancer; PSA: Prostate specific antigen; RFS: Recurrence-free survival; RP: Radical prostatectomy; SEER: The Surveillance, Epidemiology, and End Results

\section{Acknowledgments}

The authors acknowledge the efforts of the Surveillance Research Program in the establishment and maintenance of the SEER database.

\section{Authors' contributions}

$X Z, Q N, X M$ are responsible for writing, collecting data, analysis,

interpretation, revision and final approval of present article. KJ, TZ are responsible for analysis and revision. All authors have read and approved the final manuscript.

\section{Funding}

This research did not receive any specific grant from funding agencies in the public, commercial, or not-for-profit sectors.

Availability of data and materials

All analyzed data are publicly available at the SEER website (http://www.seer. cancer.gov), and should be requested under the approval of the SEER

Program administration.

\section{Ethics approval and consent to participate}

This study used a general dataset from the Surveillance, Epidemiology, and End Results database built by a public health program, and therefore did not require institutional review board approval and the patients' data were atomized.

Consent for publication

Not applicable.

\section{Competing interests}

The authors declare that they have no competing interests.

\section{Author details}

${ }^{1}$ Department of Biotherapy, West China Hospital and State Key Laboratory of Biotherapy, Sichuan University, Chengdu, People's Republic of China. ${ }^{2}$ Department of Urology, Institute of Urology and National Clinical Research Center for Geriatrics and Center of Biomedical Big Data, West China Hospital, Sichuan University, Chengdu, Sichuan Province, People's Republic of China.

${ }^{3}$ West China School of Medicine, Sichuan University, Chengdu, People's Republic of China

Received: 3 November 2019 Accepted: 21 January 2020

Published online: 04 February 2020

References

1. Siegel RL, Miller KD, Jemal A. Cancer statistics, 2019. CA Cancer J Clin. 2019; $1: 7-34$

2. Mottet N, Bellmunt J, Bolla M, et al. EAU-ESTRO-SIOG guidelines on prostate cancer. Part 1: screening, diagnosis, and local treatment with curative intent. Eur Urol. 2017;4:618-29.

3. Huland $H$, Graefen M. Changing trends in surgical management of prostate cancer: the end of overtreatment? Eur Urol. 2015;2:175-8.

4. Fletcher SA, von Landenberg N, Cole AP, et al. Contemporary national trends in prostate cancer risk profile at diagnosis. Prostate Cancer Prostatic Dis. 2019. https://doi.org/10.1038/s41391-019-0157-y.

5. Mohler JL, Antonarakis ES, Armstrong AJ, et al. Prostate cancer, version 2. 2019, NCCN clinical practice guidelines in oncology. J Natl Compr Cancer Netw. 2019:5:479-505.

6. Nagao K, Matsuyama H, Matsumoto $\mathrm{H}$, et al. Identification of curable high-risk prostate cancer using radical prostatectomy alone: who are the good candidates for undergoing radical prostatectomy among patients with high-risk prostate cancer? Int J Clin Oncol. 2018;4:757-64.

7. Ploussard G, Masson-Lecomte A, Beauval JB, et al. Radical prostatectomy for high-risk prostate cancer defined by preoperative criteria: oncologic followup in national multicenter study in 813 patients and assessment of easy-touse prognostic substratification. Urology. 2011;3:607-13.

8. Cronin KA, Ries LA, Edwards BK. The surveillance, epidemiology, and end results (SEER) program of the national cancer institute. Cancer. 2014; 120(Suppl 23):3755-7.

9. Cuccurullo V. AJCC cancer staging handbook: from the AJCC cancer staging manual (7Th edition). Eur J Nucl Med Mol Imaging. 2011;2:408. 
10. Tward JD, Kokeny KE, Shrieve DC. Radiation therapy for clinically nodepositive prostate adenocarcinoma is correlated with improved overall and prostate cancer-specific survival. Pract Radiat Oncol. 2013;3:234-40.

11. Vagnoni $V$, Bianchi $L$, Borghesi $M$, et al. Adverse features and competing risk mortality in patients with high-risk prostate cancer. Clin Genitourin Cancer. 2017;2:e239-48.

12. Weiss A, Chavez-Macgregor M, Lichtensztajn DY, et al. Validation study of the American joint committee on cancer eighth edition prognostic stage compared with the anatomic stage in breast cancer. JAMA Oncol. 2018;4: 203-9.

13. Camp RL, Marisa DF, Rimm DL. X-tile: a new bio-informatics tool for biomarker assessment and outcome-based cut-point optimization. Clin Cancer Res. 2004:21:7252-9.

14. Balachandran VP, Gonen M, Smith JJ, DeMatteo RP. Nomograms in oncology: more than meets the eye. Lancet Oncol. 2015;4:e173-80.

15. Shouval R, Labopin M, Gorin NC, et al. Individualized prediction of leukemiafree survival after autologous stem cell transplantation in acute myeloid leukemia. Cancer. 2019;125:3566-73.

16. Cho CSM, Gonen MP, Shia JM, et al. A novel prognostic nomogram is more accurate than conventional staging Systems for predicting survival after resection of hepatocellular carcinoma. J Am Coll Surgeons. 2008;2:281-91.

17. Wong SL, Kattan MW, McMasters KM, Coit DG. A Nomogram that predicts the presence of sentinel node metastasis in melanoma with better discrimination than the American joint committee on cancer staging system. Ann Surg Oncol. 2005;4:282-8.

18. Wu Y, Meyers JP, Shi G, et al. A nomogram for predicting survival and retroperitoneal lymph node dissection treatment in patients with resected testicular germ cell tumors. J Surg Oncol. 2019;3:508-17.

19. Kim BH, Kim K, Chie EK, et al. Risk stratification and prognostic nomogram for post-recurrence overall survival in patients with recurrent extrahepatic cholangiocarcinoma. Hpb. 2017;5:421-8.

20. Kim Y, Park HC, Yoon SM, et al. Prognostic group stratification and nomogram for predicting overall survival in patients who received radiotherapy for abdominal lymph node metastasis from hepatocellular carcinoma: a multi-institutional retrospective study (KROG 15-02). Oncotarget. 2017;55:94450-61.

21. Epstein Jl, Egevad L, Amin MB, Delahunt B, Srigley JR, Humphrey PA. The 2014 International Society of Urological Pathology (ISUP) consensus conference on Gleason grading of prostatic carcinoma: definition of grading patterns and proposal for a new grading system. Am J Surg Pathol. 2016;2:244-52.

22. Sundi $D$, Wang $V$, Pierorazio PM, et al. Identification of men with the highest risk of early disease recurrence after radical prostatectomy. Prostate. 2014:6:628-36.

23. Kim $\mathrm{TH}$, Jeon $\mathrm{HG}$, Jeong $\mathrm{BC}$, et al. Development of a new nomogram to predict insignificant prostate cancer in patients undergoing radical prostatectomy. Scand J Urol. 2017;1:27-32.

24. Grossklaus DJ, Coffey CS, Shappell SB, Jack GS, Chang SS, Cookson MS Percent of cancer in the biopsy set predicts pathological findings after prostatectomy. J Urol. 2002;5:2032-6.

25. Hamada R, Nakashima J, Ohori M, et al. Preoperative predictive factors and further risk stratification of biochemical recurrence in clinically localized high-risk prostate cancer. Int J Clin Oncol. 2016:3:595-600.

26. Evangelista L, Guttilla A, Zattoni F, Muzzio PC, Zattoni F. Utility of choline positron emission tomography/computed tomography for lymph node involvement identification in intermediate- to high-risk prostate cancer: a systematic literature review and meta-analysis. Eur Urol. 2013;6:1040-8.

27. Sheng $W$, Zhang $H$, Lu Y. Survival outcomes of locally advanced prostate cancer in patients aged $<50$ years after local therapy in the contemporary US population. Int Urol Nephrol. 2018;8:1435-44.

28. Danella JF, DeKernion JB, Smith RB, Steckel J. The contemporary incidence of lymph node metastases in prostate cancer: implications for laparoscopic lymph node dissection. J Urol. 1993;6:1488-91.

29. Amin MB, Greene FL, Edge SB, et al. The eighth edition AJCC Cancer staging manual: continuing to build a bridge from a population-based to a more "personalized" approach to cancer staging. CA Cancer J Clin. 2017;2: 93-9.

30. Mottet N, van den Bergh RCN, Briers E, et al. EAU Guidelines. https:// uroweb.org/guidelines/2019. Accessed 10 Jan 2020.
31. Pilepich MV, Winter K, Lawton CA, et al. Androgen suppression adjuvant to definitive radiotherapy in prostate carcinoma--long-term results of phase III RTOG 85-31. Int J Radiat Oncol Biol Phys. 2005;5:1285-90.

32. James ND, Spears MR, Clarke NW, et al. Failure-free survival and radiotherapy in patients with newly diagnosed nonmetastatic prostate cancer: data from patients in the control arm of the STAMPEDE trial. JAMA Oncol. 2016;3:348-57.

33. Stranne J, Brasso K, Brennhovd B, et al. SPCG-15: a prospective randomized study comparing primary radical prostatectomy and primary radiotherapy plus androgen deprivation therapy for locally advanced prostate cancer. Scand J Urol. 2018:5-6:313-20.

\section{Publisher's Note}

Springer Nature remains neutral with regard to jurisdictional claims in published maps and institutional affiliations.
Ready to submit your research? Choose BMC and benefit from:

- fast, convenient online submission

- thorough peer review by experienced researchers in your field

- rapid publication on acceptance

- support for research data, including large and complex data types

- gold Open Access which fosters wider collaboration and increased citations

- maximum visibility for your research: over $100 \mathrm{M}$ website views per year

At BMC, research is always in progress.

Learn more biomedcentral.com/submissions 